punto org

Collana diretta da Luigi Maria Sicca

30 
Francesco D’ERRICO

\section{ARMONIA FUNZIONALE E MODALITÀ}

RUDIMENTI PER L'IMPROVVISAZIONE

A INDIRIZZO JAZZISTICO

introduzione di

Francesco Piro

prefazione di

Roberto Grisley

Editoriale Scientifica

Napoli 
Tutti i diritti sono riservati

(C) 2017 Editoriale Scientifica srl Via San Biagio dei Librai 39 80138 Napoli www.editorialescientifica.com info@editorialescientifica.com ISBN 978-88-9391-151-1 


\section{Indice}

11 Introduzione di Francesco Piro

17 Prefazione di Roberto Grisley

25 Premessa dell'autore

29 Avvertenza dell'autore

Parte prima

\section{Rudimenti di armonia funzionale}

\section{Capitolo 1}

37 1.1. Aspettative acustiche

38 1.2. Elementi tensivi e risolutivi

38 1.3. Triadi del I, IV e V grado. Tono, semitono

40 1.4. Triadi sui sette gradi, loro funzioni e rivolti

44 1.5. Intervalli

46 1.6. Cadenze. I tabella dei percorsi armonici

49 Esercizi

51 Capitolo 2

51 2.1. Triadi maggiori minori aumentate e diminuite

52 2.2. Gli accordi derivati: II III VI VII

53 2.3. Ambiguità degli accordi del III e VI grado.

Gli accordi satellite

54 2.4. Funzioni tonali univoche e biunivoche. Le note guida

56 2.5. Sostituzioni di funzione e di colore

57 2.6. Accordi di settima

58 2.7. Profilo funzionale degli accordi di settima

59 2.8. Note guida 
59 2.9. L'accordo di settima di dominante

61 2.10. I movimenti armonici I V e V I come paradigma

62 2.11. Spostamenti di quarta e quinta e rudimenti di sostituzione

63 2.12. II tabella dei percorsi armonici

65 Esercizi

$67 \quad$ Capitolo 3

67 3.1. Tonicizzazione

68 3.2. Transizioni

68 3.3. Dominanti secondarie

69 3.4. Prospetto dominanti secondarie

71 3.5. Dominanti estetse

72 3.6. Sostituzioni al tritono

72 3.7. Dominanti e note guida

73 3.8. Ancora note guida

74 3.9. Dominanti principale e sostituzione al tritono

75 3.10. Sostituzione di funzione e di colore

76 3.11. Ancora funzione e colore

77 Esercizi

79 Capitolo 4

79 4.1. Introduzione al modo minore

79 4.2. Regione tonale

80 4.3. Regioni tonali e improvvisazione

80 4.4. Minore naturale: relativo, omologo o parallelo

81 4.5. Minore armonico e melodico

82 4.6. Triadi e accordi di settima nel modo minore

83 4.7. Funzioni tonali in minore

84 4.8. III tabella dei percorsi armonici

84 4.9. Gli accordi del minore armonico

85 4.10. Profili melodici nel minore

85 4.11. Interscambio modale

86 4.12. Cadenze principali e interscambio modale

$87 \quad$ Esercizi

\section{$87 \quad$ Capitolo 5}

87 5.1. Altre qualità dell'accordo di dominante

88 5.2. L'accordo di settima diminuita 
90 5.3. Accordi di settima diminuita e dominanti

91 5.4. Dominanti: quattro direzioni

92 5.5. Quattro accordi di dominante

93 5.6. Dominanti funzionali e dominanti non funzionali

94 5.7. Gradualità di cadenza delle quattro dominanti

95 5.8. Le dominati non funzionali secondarie ed estese

95 5.9. Triadi aumentate

96 5.10. Triadi aumentate e dominanti

98 5.11. Dodici accordi di dominante, una tonica

99 5.12. Gradualità di cadenza dei dodici accordi di dominante

100 5.13. Sottodominanti

100 5.14. Sottodominanti secondarie ed estese

101 5.15. Le dodici sottodominanti e sostituzione al tritono

102 Esercizi

105 Capitolo 6

105 6.1. Regioni tonali e scale

106 6.2. Maggiore e minore: reciprocità

107 6.3. Tonalità vicine e lontane

108 6.4. Differenti tonalità, triadi condivise

108 6.5. Modulazioni ai toni vicini

110 6.6. Modulazioni ai toni lontani

111 6.7. Significato acustico delle triadi nelle modulazioni

112 6.8. Modulazioni e modo minore

113 6.9. Modulazione e interscambio modale sul II e IV grado

115 6.10. Modulazione e interscambio modale

116 6.11. Appendice A: Circolo delle quinte e derivati

119 6.12. Appendice B: Tournaround

122 6.13. Tournaround e sottodominanti

123 6.14. Tritonic system

124 Esercizi

Parte seconda

\section{Rudimenti del sistema modale}

129 Capitolo 7

129 7.1. Dalle gerarchie cadenzali all'orizzonte dei modi

130 7.2. I soli modi del '900 occidentale 
130 7.3. I modi generati dalla scala maggiore

133 7.4. Il carattere dei modi declinazioni emotive e geografiche

134 7.5. I modi generati dalla scala maggiore armonica e da quelle minori melodica e armonica

139 Esercizi

\section{Capitolo 8}

141 8.1. Gradi e modi

142 8.2. I modi ordinati per terze

144 8.3. Modi sugli accordi del V grado

146 8.4. Modi sugli accordi del II grado

147 8.5. Modi e funzioni tonali: per una metodologia

147 8.6. Le scale simmetriche

148 8.7. Le scale diminuite o ottofoniche

149 8.8. Scale di sei suoni

149 8.9. Le scale aumentate

150 8.10. Le scale esatonali

151 8.11. Tabella delle relazioni modo/accordo

154 Esercizi

\section{Capitolo 9}

157 9.1. Il sistema modale come modello autonomo

157 9.2. Le cadenze modali

158 9.3. Tre cadenze modali

159 9.4. Un esempio di cadenza dorica (So What voicing). Pentatoniche

161 9.5. Un esempio di cadenza Ionica

162 9.6. Un esempio di cadenza frigia

164 Esercizi

\section{Capitolo 10}

165 10.1. I confini del sistema modale

165 10.2. Fuori dalle funzioni tonali

166 10.3. Semitono e percezione nei modi

168 10.4. Due modelli espressione dal sistema modale

169 10.5. Modi giustapposti: una tabella di esperienza acustica

169 Esercizi 


\section{Capitolo 11}

173 11.1. Modale e interscambio modale

173 11.2. Accordi di settima generati dai ventotto modi

178 11.3. Sintesi degli accordi di settima per ciascun grado

179 11.4. Estensione dell'interscambio modale:

prima procedura

180 11.5. Estensione dell'interscambio modale:

seconda procedura

182 11.6. Alternanza di due modi/un sol modo: due esempi

184 11.7. Scala Flamenco

185 11.8. Accordi derivati dalla scala flamenco

188 Esercizi

189 Conclusioni dell'autore

191 Indice tematico

195 Indice delle meditazioni

197 Bibliografia didattica

199 Indice dei nomi

201 Hanno scritto nella collana punto org 



\section{Introduzione}

Francesco Piro*

Questo libro è dedicato a uno dei temi che attraversa la rete internazionale di ricerca puntOorg: l'esplorazione e l'analisi interna alla musica come forma di attività umana organizzata. Si tratta di un testo tecnico in senso ampio: "rudimenti" (in tedesco si scriverebbe Anfangsgründe, Grundlinien o forse Grundrisse) dell'armonia e della modalità, per chi voglia capire davvero le logiche dell'improvvisazione musicale (in primo luogo jazzistica), al fine di meglio praticarla. Si tratta soprattutto di un libro scritto da un musicista - eccellente, me lo si consenta - e rivolto a quanti debbano apprendere l'arte di improvvisare, con esempi tratti dalla straordinaria esperienza novecentesca del jazz.

La musica è divenuta una costante in puntOorg, a partire dall' intuizione (e dal diretto impegno di tessitura intellettuale) del direttore scientifico, Luigi Maria Sicca, con il supporto di un folto gruppo di studiosi che vi opera piuttosto stabilmente ${ }^{1}$.

Cerchiamo di ricapitolare come ciò sia avvenuto.

Inizialmente, il progetto puntOorg (e la Collana che ne accoglie i risultati) aveva l'obiettivo già ambizioso, ma comunque ben definibile sotto il profilo accademico, di ampliare la formazione degli studiosi universitari, in particolare quelli destinati a praticare i "saperi manageriali". Si partì dalle potenzialità di un sano meticciato tra discipline diverse, finalizzato a rendere in-

*Professore ordinario di Storia della Filosofia, Università degli Studi di Salerno.

${ }^{1}$ http: / / www.puntoorg.net/it/ ricerche-e-studiosi / volumi. 
telligibile la complessità interna del mondo delle organizzazioni formali, di cui quelle aziendali rappresentano un fondamentale sotto-insieme. A questo tema era dedicato il n. 1 della Collana ${ }^{2}$, silloge che offriva strumenti di analisi e interpretazione dell'agire organizzativo, partendo da discipline come l'etnologia, gli studi culturali, la narratologia. Chi scrive lavorò a quel progetto sin dall'inizio, insieme al curatore, curando la "cassetta degli attrezzi" per il lettore ${ }^{3}$.

L'obiettivo fondamentale della Collana, allora come ora, era dunque di potenziare la ricerca scientifica sul mondo economico-organizzativo. All'interno di questo obiettivo di fondo, vi era tuttavia in nuce anche un' esigenza di riflessione sulla formazione e sui suoi nodi attuali che si manifestò ancor più chiaramente nel secondo volume, curato dal solo Sicca ${ }^{4}$ e nato come prolusione tenuta presso l'Università degli Studi del Sannio per l'inaugurazione dell'anno accademico 2010-2011 del Conservatorio di Benevento ${ }^{5}$. La questione della "testualità" si espandeva ora a tematizzazione della testualità in musica e del modo in cui i processi di lettura e interpretazione del testo divengono azione organizzativa. Fin qui sembrerebbe trattarsi semplicemente di un'estensione degli obiettivi iniziali a un diverso tipo di organizzazione. Ma vi si avverte implicitamente una svolta, grazie alla centralità che la musica vi prende come esperienza "formativa", ovvero come una di quelle "risorse" che le culture pre-capitalistiche mettono a disposizione per le nuove generazioni che dovranno individuare le vie di uscita dai circoli vi-

${ }^{2}$ L.M. Sicca (a cura di), Leggere e scrivere organizzazioni. Estetica, umanesimo e conoscenze manageriali, Napoli, Editoriale Scientifica, 2010.

${ }^{3}$ F. Piro, "Quanto umanisti dobbiamo essere ancora? Una cassetta degli attrezzi per il lettore", in Leggere e scrivere organizzazion, cit., 2010, 215240.

${ }^{4}$ L.M. Sicca, Alla fonte dei saperi manageriali. Il ruolo della musica nella ricerca per l'innovazione e per la formazione delle risorse umane, Napoli, Editoriale Scientifica, 2012.

${ }^{5}$ Il titolo di quella prolusione, riprendendo il secondo verso dell' Ars Poetica di Paul Verlaine era "E per questo preferisci il Dispari". 
ziosi della grande crisi del capitalismo contemporaneo. Non si trattava più soltanto di "applicare" alla realtà contemporanea, alle aziende come anche ad altre organizzazioni formali, un insieme di saperi "umanistici" che aiutassero a focalizzarne meglio i nodi problematici. Si trattava anche di fare i conti con alcuni saperi "esemplarmente" formativi, per capire come essi potessero offrire schemi e modelli di un agire rigoroso ma anche creativo, da estendere e generalizzare anche alle organizzazioni emergenti. E a partire di qui nasceva, tra l'altro, il progetto di costruire esperimenti che aiutassero a esibire al meglio queste stesse doti e competenze. Ricerca inter-disciplinare e innovazione intra-disciplinare entrarono in una sorta di competizione reciproca e di continuo rinvio.

Nacque un terzo volume, la raffinata riflessione di Agostino Di Scipio sul suono nelle età delle tecnologie e sul modo in cui esso sa "farsi musica" ${ }^{6}$. Il sesto volume riprendeva in chiave di "antropologia delle organizzazioni" le categorie di Mauss sul dono, in un'opera collettiva di amplissimo spettro teorico curata ancora da Sicca ${ }^{7}$. Nel n. 10 della Collana ${ }^{8}$, Rosario Diana proponeva invece una sperimentazione ardita e originalissima, un "libro-con-disco", che mette in dialogo filosofia e musica con due percorsi sincronizzati di "metabolizzazione" (se mi è consentita quest'immagine) dell'opera glaciale e geniale di Beckett. Ancora, nel n. 28 della Collana, si ripropone il modello accoppiato testo / spartito per seguire le tracce del concetto di "riso-

${ }^{6}$ A. Di Scipio, Pensare le tecnologie del suono e della musica, Napoli, Editoriale Scientifica, 2013.

${ }^{7}$ L.M. Sicca (a cura di), I linguaggi dell'organizzare. Musica e testo tra dono e disinteresse, Napoli, Editoriale Scientifica, 2014, con scritti di Umberto di Porzio, Rosario Diana, Agostino Di Scipio, Mariella De Simone, Renato Musto, Bernardo Maria Sannino, Chiara Mallozzi, Lorenzo Pone, Giancarlo Turaccio, Luigi Maria Sicca.

${ }^{8}$ R. Diana, Disappartenenza dell'Io. Filosofia e musica verso Samuel Beckett, Napoli, Editoriale Scientifica, 2016, prefazione di Luigi Maria Sicca.

${ }^{9}$ R. Diana, L.M. Sicca, G. Turaccio, Risonanze. Organizzazione Musica Scienze, Napoli, Editoriale Scientifica, 2017, prefazione di Antonio Strati, postfazione di Alessandro Solbiati; con contributi, ancora, di Davide Bizjak, 
nanza" tra economia, scienza, filosofia e, appunto, musica9 ${ }^{9}$ Ed è in gestazione in questi mesi una ricerca collettiva su Mario Bertoncini e la "contemporaneità", che è appunto una riflessione sull'innovazione e sul modo di leggerla e praticarla ${ }^{10}$.

Beninteso questo lavoro di riflessione dall'interno dei linguaggi delle arti punteggia una rete di ricerca (e una Collana) che resta prevalentemente dedicata all'analisi dei fondamentali problemi economici e organizzativo-aziendali del nostro tempo. Ma che un approccio di questo tipo apra uno spazio costante di approfondimento ai processi di innovazione interni alla trasmissione dei saperi è appunto un segno peculiare e caratteristico della scommessa puntOorg: non difendere con gridi tradizionalistici i saperi "umanistici" ma esplorarne le risorse e l'aiuto che possono darci a modificare l'agire. Scommessa in cui rientra, per dirlo solo cursoriamente, anche la ricerca su come sfruttare meglio di quanto non si faccia oggi il potenziale "critico" e "formativo" delle ore scolastiche e universitarie di filosofia, ricerca alla quale chi scrive ha inteso dare un contributo con la mossa paradossale di scrivere il "manuale" (cioè la mappa) di una disciplina ancora a venire, ovvero di una disciplina non-disciplina, l'educazione al pensiero critico $^{11}$. puntOorg vi tornerà a breve con ulteriori interventi, più corali ma soprattutto più concretamente legati a esperimenti di riorganizzazione dall'interno della didattica delle discipline scolastiche. Ci stiamo lavorando.

Segnalo infine che tra questi contributi in aree differenti esistono poi sistematici e costanti momenti di confronto. Ricordo per esempio la tavola rotonda su "Pensiero creativo e

Dario Casillo, Rosario Diana, Umberto di Porzio, Agostino Di Scipio, Chiara Mallozzi, Mario Nicodemi, Lorenzo Pone, Rosalba Quindici, Sonia Ritondale, Tommaso Rossi, Bernardo Maria Sannino, Luigi Maria Sicca, Cristian Sommaiuolo, Giancarlo Turaccio e Paolo Valerio.

${ }^{10}$ A cura di Chiara Mallozzi e Daniela Tortora.

${ }^{11}$ F. Piro, Manuale di educazione al pensiero critico. Comprendere e argomentare, prefazione di Tullio De Mauro, Napoli, Editoriale Scientifica, 2015. 
pensiero critico. Confronto per una formazione in tempi di crisi" che si tenne a Napoli, presso la sede dell'Editoriale Scientifica il 13 maggio 2016 con il contributo di Francesco D'Errico, dello scrivente, di Paolo de Vita, Mauro Maldonato, Cristina Mele, Mario Nicodemi, Anna Palmieri, Massimo Squillante, Daniela Tortora, Paolo Valerio, con il coordinamento di Luigi Maria Sicca. Con molti di questi studiosi esiste ormai un consolidato rapporto di confronto sui temi puntOorg tra i quali vorrei ricordare qui quello interno e intorno al mondo transgender, al centro di un altro tipo di "trasformazione di pratiche" che puntOorg studia e cerca di favorire ${ }^{12}$.

I due volumi di Francesco D'Errico si iscrivono a pieno titolo in questa feconda "deriva" che dalla riflessione sull'agire organizzativo e sulle sue forme tipiche ha portato alle esperienze-pilota di una didattica che orienti diversamente l'apprendimento e l'agire. In un precedente contributo, D'Errico discuteva dell'improvvisazione come una forma di decision making, trattandone in termini fenomenologici, filosofici, linguistici (con un impegnativo confronto tra costruzione narrativa e costruzione musicale $)^{13}$, per aiutarci a fare i conti con lo strano fatto che per l'essere umano sia possibile, piacevole, addirittura cognitivamente indispensabile, l'improvvisare.

Tra quell'opera, di pregio anche per la qualità della scrittura, e codesta che il lettore ha in mano vi sono sottili, ma evi-

${ }^{12}$ Due almeno le ricerche da segnalare: 1) S. Oliverio, L.M. Sicca, P. Valerio, Trasformare le pratiche nelle organizzazioni di lavoro e di pensiero, Napoli, Editoriale Scientifica, 2015; prefazione di Gaetano Manfredi, con la partecipazione, tra gli altri, di Davide Bizjak, Ilaria Boncori, Rossella Bonito Oliva, Guglielmo Faldetta, Vittoria Fiorelli, Edoardo Mollona, Luca Solari; 2) I. Boncori (ed.), LGBT+ perspectives. The University of Essex Reader, Napoli, Editoriale Scientifica, 2017.

${ }^{13}$ F. D'Errico, Fuor di metafora. Sette osservazioni sull'improvvisazione musicale, prefazione di Paolo de Vita, Postfazione di Mauro Maldonato, Napoli, Editoriale Scientifica, 2015. 
denti fili di continuità. Non che D'Errico ritenga che la prassi improvvisativa possa essere prefigurata o organizzata dalla conoscenza teorica. D'Errico, anzi, lo nega con forza. Ciò che la teoria - o piuttosto la meditazione - può fare, è soprattutto "liberare" la prassi da precostruzioni teoriche tradizionali, permettendole di concentrarsi tutta sulle possibilità intrinseche dell'"oggetto sonoro" che viene direttamente sperimentato dall'improvvisatore.

Con un tono memore del grande valore liberatorio che ebbe la filosofia fenomenologica nel Novecento, D'Errico chiede dunque una percezione concentrata sull'accadimento musicale, capace di "non privilegiare nessun punto di partenza", massima, quest'ultima, che forse potrebbe riassumere l'intero libro.

Vi è però anche un'altra parola-chiave che ritroviamo nel libro e mi sembra chiarificante, "opportunità": l'esperienza diretta e partecipativa del suono è soprattutto la percezione di un'opportunità di prosecuzione, la percezione immediata di quella che con Leibniz (altro autore caro a D'Errico) potremmo definire una "possibilità".

La chiarezza del linguaggio, l'alternanza tra l'esposizione di conoscenze e la proposta di esercitazioni mirate che le consolidano, il rinvio discreto ai possibili approfondimenti, fanno di questo libro un capolavoro didattico in pieno stile puntOorg, invidiato anche da chi, come lo scrivente, una nuova didattica la sta proponendo in altri àmbiti.

Ancora una volta, si riscontra qui quello strano valore esemplare, prefigurante, che ha la musica, non solo rispetto alle altre "tecniche", ma rispetto alla "prassi" umana in genere. Di questo valore siamo consci e cerchiamo di trasmetterlo a un XXI secolo ancora in bilico tra tecno-barbarie e tecno-cultura.

Salerno, Università degli Studi

6 Maggio 2017 